\title{
Design and Functional Evaluation of an Epidermal Strain Sensing System for Hand Tracking
}

\author{
Hadrien O. Michaud ${ }^{1}$, Laurent Dejace ${ }^{1}$, Séverine de Mulatier ${ }^{1}$ and Stéphanie P. Lacour ${ }^{1}$
}

\begin{abstract}
This study demonstrates soft, epidermal resistive strain gauges capable of tracking finger joint angle during dexterous manipulation tasks. Intrinsically stretchable, biphasic, gallium-based metal films embedded in an elastomeric substrate allow for extremely thin $(<\mathbf{5 0} \mu \mathrm{m})$ and skin-conforming wearable sensors with outstanding robustness. The sensors sustain repeated cycling to $50 \%$ strain and are insensitive to normal pressure up to $100 \mathrm{kPa}$. Following a calibration phase, we recorded flexions of a human finger using the soft sensors and compared their joint angle estimation to that of a commercial marker-based visual motion tracking system. The accuracy of our system (defined as the mean angular deviation between our sensors' output and the reference system) was below $9^{\circ}$ over a set of 11 different grasping and motion tasks. We demonstrate the scalability and wearability of our technology with a threefinger sensing system used to track the fine movements of a pianist hand. Our soft technology is a promising candidate for implementation of truly wearable proprioceptive sensing systems.
\end{abstract}

\section{INTRODUCTION}

Trauma and neurological disorders often impair mobility and natural control of the upper limbs. Therapies designed to help patients improving and ultimately regaining motor control often implement robotic technologies. While "wearable actuation", e.g. in the form of exoskeleton, becomes available, their "wearable sensing" counterpart remains challenging to integrate reliably on the body. Simple, wearable systems capable of tracking movement of small body parts such as the hand are critical to assess that patients are performing correct therapeutical movements and quantify their progress. Vision or robotic based systems are widely used in research or clinical therapy to provide high accuracy in movement reconstruction. Their use may promote more effective and faster rehabilitation [1]. However, they require complex, large and/or expensive equipment and software for data treatment that are difficult to adapt to a system for daily usage in clinic and at home. Inertial sensors are easier to integrate into a portable system but are prone to integration drift, which limits their accuracy over long periods of time [2]. Data gloves are a wearable set-up alternative to monitor hand motion and provide positional feedback during rehabilitation therapies or assistive devices usage [3], [4]. However, commercially available systems such as the Data Glove (5DT Inc. Irvine, CA) or the Cyberglove (Cyberglove Systems LLC.

*This work was supported by the Fondation Bertarelli, nano-tera.ch (20NA 143070 WiseSkin) and the Swiss National Science Foundation through the National Centre of Competence in Research (NCCR) in Robotics.

${ }^{1}$ H.O. Michaud, L. Dejace, S. de Mulatier and S.P. Lacour are with the Laboratory for Soft Biolectronic Interfaces, Center for Neuroprosthetics, EPFL, Lausanne, Switzerland stephanie.lacour at epfl.ch
San Jose, CA) are cumbersome and often restrict the natural motion of the fingers. In the neurorehabilitation context, gloves may also impede access to muscle groups, limiting their availability for recording and stimulation. Moreover, they cover the hand palm, altering tactile sensation and grip ability. After a stroke, hand palsy may occur, leaving the hand locked in a closed fist position; tracking gloves then become difficult if not impossible to be put on. Recent approaches intending to improve the wearability of motion sensing devices focus on combining soft material carriers with stretchable conductive materials. Elastomers display skin-like properties and engineering elastic conductors with high electromechanical performance is an active field of research [5]-[8].

A variety of transducers including capacitive, resistive and piezoresistive sensors, has been integrated in wearable skins. Resistive transducers require a single conductive layer embedded in a polymer carrier; they display low sensitivity to electromagnetic or contact parasitic interferences and can be interfaced with a wide variety of circuits, enabling miniaturization and portability of sensor arrays. Capacitive sensors are often more stable and linear but at the expense of a multi-layered structure including effective shielding [9].

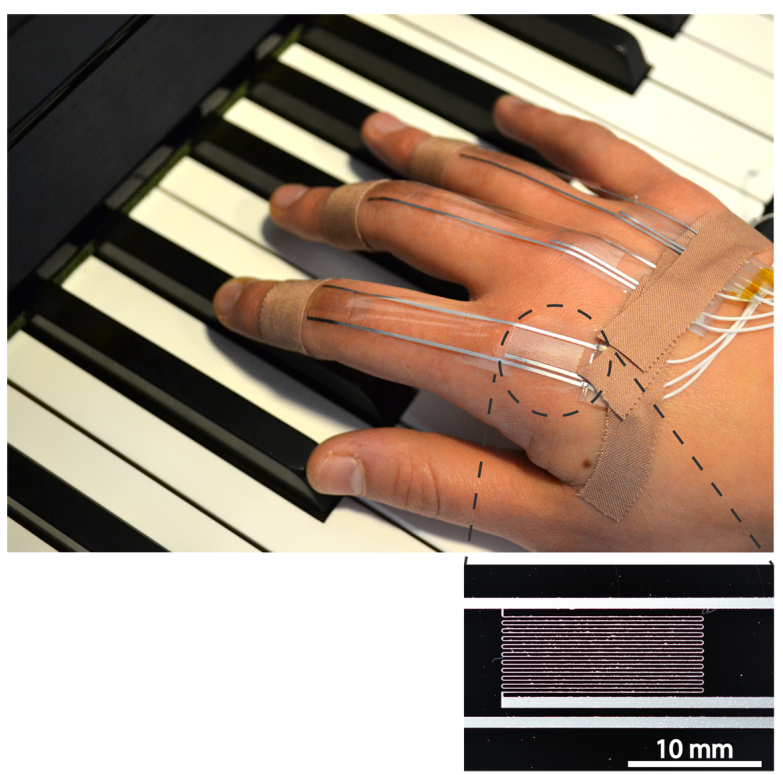

Fig. 1. A system composed of three sensing strain gauges is placed on a hand performing a fine motor task. The gauge terminal tracks are $800 \mu \mathrm{m}$ wide and the meander pattern is composed of $50 \mu \mathrm{m}$ wide tracks forming 20 parallel tracks. 
Soft resistance sensors may be positioned over the moving joints of the finger. Designs including thin films with high strain sensitivity [10], highly resistive ionic liquids [11], and highly resistive composites [12] have been reported. Another approach consists in translating the design of traditional foil strain gauges to elastic carrier substrates, and preparing the sensitive meanders with stretchable conductive materials [13], [14].

Here, we report on soft strain sensors fabricated using a novel metallization technology of intrinsically stretchable biphasic thin metal films deposited and micro-structured on elastomeric substrates [15]. This method enables a new type of extremely conformal, soft (Young's modulus $<2 \mathrm{MPa}$ ) and thin (thickness $<50 \mu \mathrm{m}$ ) resistive strain gauges that can stretch up to more than $50 \%$ strain while remaining electrically conductive. Overall, the thickness and mechanical stiffness of these sensors mimic the physical properties of the stratum corneum, the outermost layer of the skin (10 $\mu \mathrm{m}$ to $40 \mu \mathrm{m}$ in thickness and Young's modulus of $6 \mathrm{MPa}$ ) [16]. Compared to solutions based on micro-channels filled with conductive liquids, our proposed gauges are one to two orders of magnitude thinner [11], [14]. Their form factor makes them unobtrusive when worn on the skin.

We demonstrate that our strain gauges behave similarly to traditional strain gauges even at very large strains, and that their performance is minimally influenced by transverse strains or normal pressure. We show that they can be used as flexion sensors with sensitivity greater than $7 \times 10^{-4}$ $/ \mathrm{deg}$ (equivalent to about $5 \Omega / \mathrm{deg}$ for a sensor having an initial resistance of $7 \mathrm{k} \Omega$ ). As a proof of concept, we placed pairs of strain gauges on a human hand performing different types of grasps and tasks. After calibration, we recorded the motions of one or several fingers with minimal movement impediment, and even when performing fine motor tasks such as playing the piano (Fig. 1). We compared the measurement of the soft sensors to the ones obtained with a commercial video-based motion tracking system and assessed their repeatability, accuracy, dynamic characteristics and stability over a panel of eleven tasks. In addition, the thin sensors were robust and could be manipulated repeatedly, as well as mounted and adjusted to form an unobtrusive sensing system covering the surface of the hand.

\section{SENSORS DESIGN AND FABRICATION}

A. First design: sensors for characterization under uniaxial strain

The size of the sensors was selected in order to fit in our customized linear stretcher. The sensors featured large terminal tracks (800 $\mu \mathrm{m}$ width) and thin meander patterns (sixteen $100 \mu \mathrm{m}$ wide parallel tracks, Fig. 2-left).

\section{B. Second design: on-finger flexion and extension sensors}

Long sensing strips $\left(80 \times 9.8 \mathrm{~mm}^{2}\right)$ hosted two flexion and extension sensors (Fig. 2-right). Each sensor consisted of two meander patterns (sixteen $100 \mu \mathrm{m}$ wide parallel tracks or twenty $50 \mu \mathrm{m}$ wide parallel tracks) interconnected to $800 \mu \mathrm{m}$

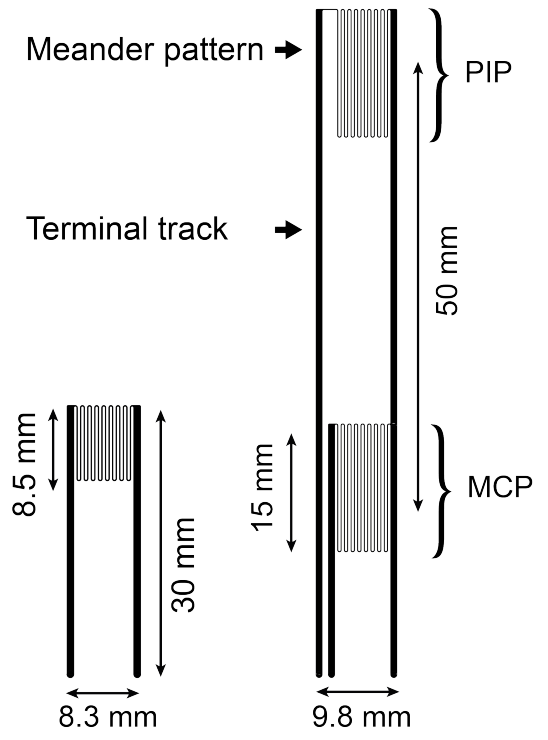

Fig. 2. Design of a single sensor for characterization (left) and sensor pair for on-finger flexion and extension sensing (right). Meander patterns were composed of sixteen $100 \mu \mathrm{m}$ wide parallel tracks for the single sensor and sixteen $100 \mu \mathrm{m}$ or twenty $50 \mu \mathrm{m}$ wide parallel tracks for the sensor pair for on-finger flexion sensing. Terminal tracks were $800 \mu \mathrm{m}$ wide for both designs.

wide terminal tracks. We chose the spacing $(50 \mathrm{~mm}$ center to center) and length $(15 \mathrm{~mm})$ of the gauges to cover most of the human finger length [17]. Assuming a constant sheet resistance, the meander patterns are at least 35 times more resistive than the terminal tracks for all sensor pairs designs. Hence, most of the sensed signal comes from the meanders and the cross talk from the tracks is minimized. Two sensors covering the metacarpophalangeal (MCP) and proximal interphalangeal (PIP) joints are sufficient to reconstruct the whole finger movement since the movement of the distal interphalangeal joint (DIP) is strongly coupled to the PIP joint [18].

\section{Fabrication}

The fabrication process is summarized Fig. 3. A support silicon wafer was treated with a self assembled layer of trichloro $(1 \mathrm{H}, 1 \mathrm{H}, 2 \mathrm{H}, 2 \mathrm{H}$-perfluorooctyl)silane (SigmaAldrich). A $25-\mu \mathrm{m}$-thick polydimethylsiloxane (PDMS, Sylgard 184, Dow Corning) film was prepared by mixing and then spin-coating the elastomer base and its curing agent at a 10:1 weight ratio on the support wafer. After curing for $2 \mathrm{~h}$ at $80{ }^{\circ} \mathrm{C}$, the PDMS surface was treated by oxygen plasma for $30 \mathrm{~s}$ at $29 \mathrm{~W}$. Photoresist (AZ9260, AZ Electronic Materials) was then spin-coated at 6000 rpm for $90 \mathrm{~s}$ and left to dry overnight. It was exposed with $220 \mathrm{~mJ} / \mathrm{cm}^{2}$ UV light (MJB4, SUSS MicroTech) through a photolithography chromium mask and developed for $90 \mathrm{~s}$ in $\mathrm{AZ} 400 \mathrm{~K}$ diluted in DI water at a 1:5 vol. ratio. Subsequently, a 40-nm-thick Au adhesion layer was sputtered on the PDMS (DP 650, Alliance-Concept) and 0.2 $\mathrm{g}$ of $\mathrm{Ga}$ were thermally evaporated (VACO 250, Vacotec) 
(a)

1. PDMS spin coating

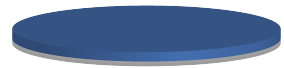

3. Au/Ga deposition

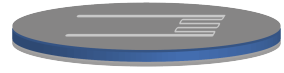

2. Photoresist patterning

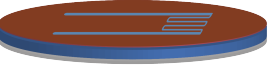

4. Lift-off

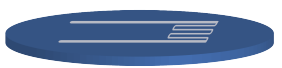

(b)

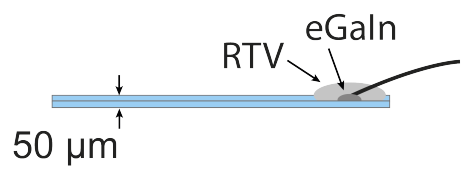

Fig. 3. (a) Process flow for the microfabrication of the soft sensors. (b) Cross-section of a sensor connected with external wiring.

to form a solid-liquid biphasic film. More details on the stretchable thin film deposition and characterization can be found in [15]. The photoresist was removed by lift-off in SVC-14 (Shipley) for $24 \mathrm{~h}$. Next, the metallized tracks were connected to PTFE stranded wires (Habia) with eutectic gallium-indium (EGaIn, Sigma-Aldrich) and encapsulated with a silicone sealant (734, Dow Corning). The sensors were encapsulated by spin-coating a second $25-\mu \mathrm{m}$-thick PDMS layer. They were finally cut and manually peeled off the support wafer before usage.

\section{RESPONSE TO UNIAXIAL STRAIN, NORMAL FORCE, AND TEMPERATURE VARIATION}

\section{A. Experimental set-up and instrumentation}

For uniaxial strain characterization, we applied a controlled displacement using a custom horizontal stretching apparatus. Displacement was driven using a rotary motor (BMS60-UFA, Aerotech) and encoded by two inductive position sensors (Li200P0-Q25LM0, Turck). The sensors were held by $40 \mathrm{~mm}$ wide clamps, as shown Fig. 4 .

For pressure response testing, we applied a controlled normal force on the sensors over an area of $23 \times 14 \mathrm{~mm}^{2}$ using an electromechanical Universal Test System (Criterion C.42, MTS) with a $100 \mathrm{~N}$ load cell.

For thermal response testing, we placed the sensors in a temperature controlled oven (UFE 500, Memmert), and monitored the resistance of the sensor while temperature was raised from room temperature to $100{ }^{\circ} \mathrm{C}$.

\section{B. Results}

The gauge factor in the $\mathrm{x}$-direction is 1.2 whereas it is only 0.05 in the y-direction (Fig. 4a-b). The strain gauges are hence 24 times more sensitive to strains in the designed sensing direction than in the transverse direction. This selective sensitivity is a key feature for strain gauges [19].

Sensitivity to normal pressure is less than 0.2 /MPa in the 0 to $100 \mathrm{kPa}$ pressure range (Fig. $4 \mathrm{c}$ ). (a)

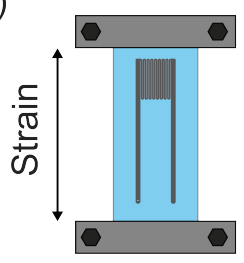

(b)
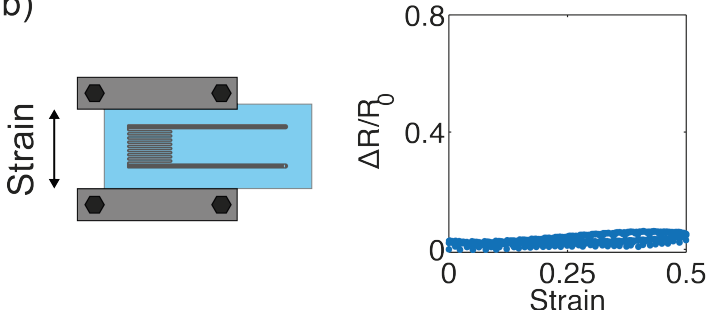

(c)
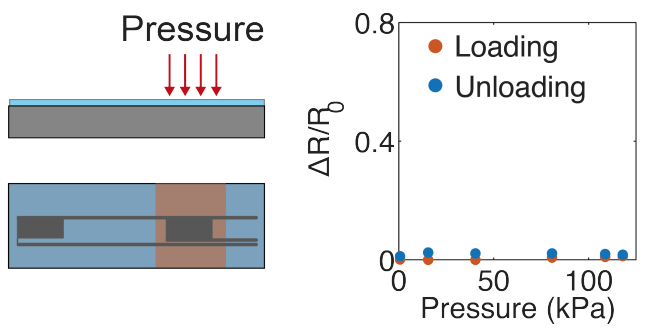

Fig. 4. Relative change in resistance of a characterization sensor as a function of (a) uniaxial longitudinal strain for 200 cycles to $50 \%$ strain, (b) uniaxial transverse strain for 20 cycles to $50 \%$ strain, (c) normal pressure.

The sensor's resistance increased linearly as a function of temperature in the range we investigated $\left(\mathrm{R}^{2}>0.99\right)$, with a sensitivity of $1.7 \times 10^{-3} /{ }^{\circ} \mathrm{C}$.

\section{BENCHMARKING AS FINGER TRACKING SENSORS}

\section{A. Experimental set-up}

The MCP and PIP angles computed by the sensors were compared to the angles measured by a Vicon Nexus motion tracking system (Vicon Motion Systems Ltd.), consisting of eight Bonita cameras and one data acquisition device (Vicon Datastation ADC Patch Panel, Oxford Metrics).

The sensors' resistance were recorded at $1 \mathrm{kHz}$ with a 16 bit resolution via a voltage divider. A constant input voltage of $5 \mathrm{~V}$ (2400 SourceMeter, Keithley) was applied across each track on which two $1.5 \mathrm{k} \Omega$ reference resistances were mounted. The sensing strip and the markers for the camera motion tracking were placed as depicted Fig. 5. Placement of the markers enabled the projection of the finger angles in the sagittal plane of the hand [20]. The sagittal plane was defined as perpendicular to the plane containing the $\mathrm{CMC} 2, \mathrm{MCP} 2$ and MCP3 markers. For recording with an array of three sensing strips, the signal outputs from three pairs of sensors positioned on the index, middle and ring fingers were readout using voltage dividers connected to the analog inputs of an Arduino Uno board. 
(a)

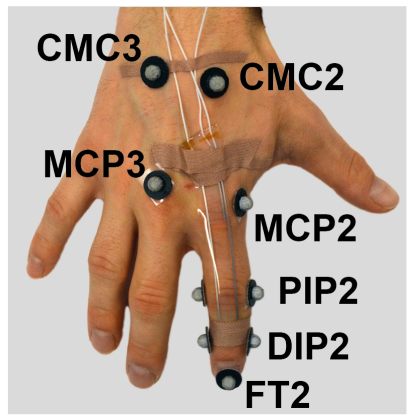

(b)

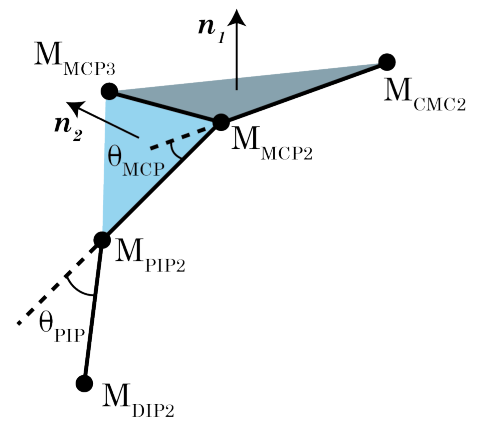

Fig. 5. (a) Camera tracking markers and sensor strip placement and attachment on the hand. (b) Definition of the MCP and PIP angles, and the plane used to compute them.

\section{B. Calibration procedure}

At the beginning of each data acquisition session, the subject was asked to sequentially flex and extend his/her MCP joint three times, then his/her PIP joints three times, then both joints three times.

From the calibration run, we applied the least squares method to determine the coefficients of a second order polynomial regression model:

$$
\begin{array}{r}
\theta=a_{0}+a_{1} \frac{\Delta R_{M C P}}{R_{0, M C P}}+a_{2} \frac{\Delta R_{P I P}}{R_{0, P I P}}+a_{12} \frac{\Delta R_{M C P}}{R_{0, M C P}} \frac{\Delta R_{P I P}}{R_{0, P I P}} \\
+a_{11}\left(\frac{\Delta R_{M C P}}{R_{0, M C P}}\right)^{2}+a_{22}\left(\frac{\Delta R_{M C P}}{R_{0, M C P}}\right)^{2} \quad \text { (1) }
\end{array}
$$

The $a_{i}$ coefficients were determined for each angle (MCP and PIP) and used to reconstruct the angles $\theta_{M C P}$ and $\theta_{P I P}$ from the resistances of the pair of sensors in every other run of the session.

We also verified that abduction and adduction of the index were minimally influencing the output signal $(1.6 \%$ of relative resistance variation over a full ad-abduction cycle) of the MCP sensor, validating the choice of studying the flexion of the finger in the sagittal plane of the hand only.

\section{Description of data acquisition sessions}

In total, three data acquisition sessions were performed, using different sensor designs or attachment strategies. The first featured $100 \mu \mathrm{m}$ wide tracks in the meander patterns, and were glued directly to the finger using skin adhesive
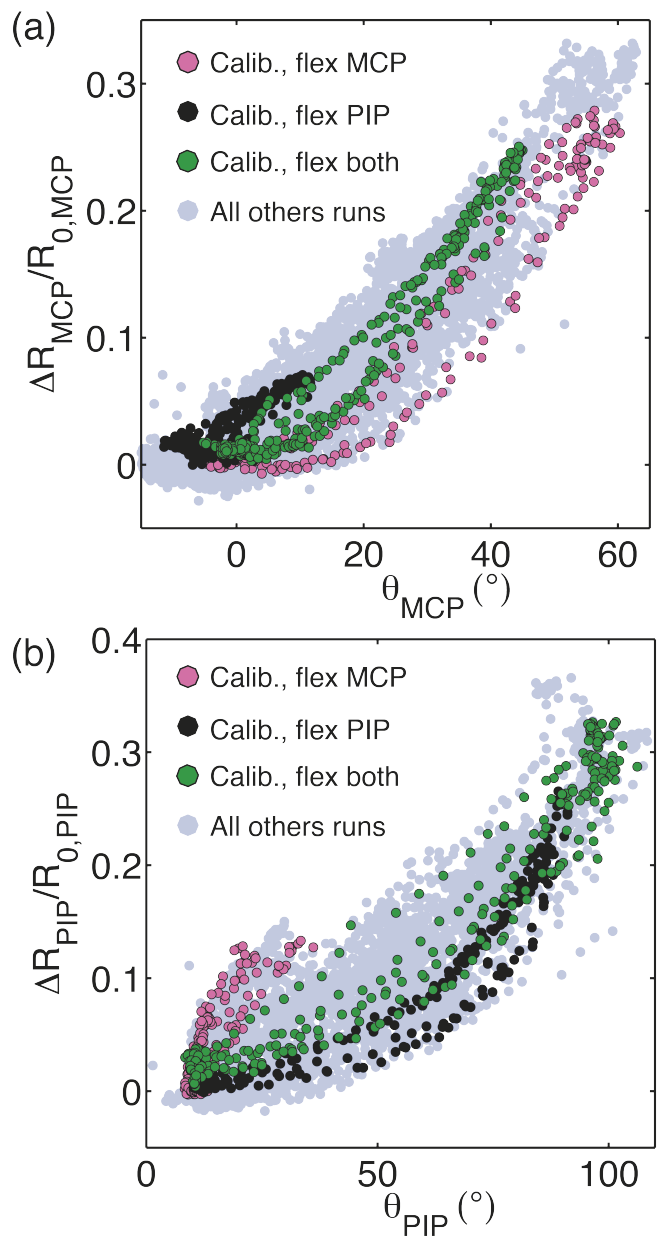

Fig. 6. (a) Relative change in resistance of the MCP sensor as a function of $\theta_{M C P}$ for the nine calibration flexions and extensions of the third session. The blue points represent the data acquired during all the other runs of the session. (b) Relative change in resistance of the PIP sensor as a function of $\theta_{P I P}$ for the nine calibration flexions and extensions of the third session. The blue points represent the data acquired during all the other runs of the session. For clarity, only one in ten points were plotted.

silicone (7-9700, Dow Corning). The second and third sessions were performed by attaching the strip to the finger with medical tape, as shown Fig. 5. Width of the meander patterns was decreased to $50 \mu \mathrm{m}$ in the third session. The use of tape enabled quicker and easier adjustment and repositioning of the strips at the beginning of the session and ensured that the sensors were slightly under tension in the resting position. Adjusting the sensors in this way prevented the apparition of a "dead-zone" in-which no signal variation occurred for low MCP or PIP angles. The whole set of grasps performed during the different sessions consisted in moving the MCP joint only, moving the PIP joint only, moving both joints, performing a power grip grasp on cylindrical objects with diameters of $65 \mathrm{~mm}, 53 \mathrm{~mm}, 39 \mathrm{~mm}$ and 28 $\mathrm{mm}$, performing a tip pinch grasp, performing a writing tripod grasp, manipulating pliers, and catching a falling bottle of water. Some examples of grasps are shown in the supplementary video and Table I details the number and types of tasks for each session. 
TABLE I

TYPE AND NUMBER OF TASKS FOR ALL DATA ACQUISITION SESSIONS

\begin{tabular}{|c||c|c|c|}
\hline & $100 \mu \mathrm{m}$, glue & $100 \mu \mathrm{m}$, tape & $50 \mu \mathrm{m}$, tape \\
\hline Flex. MCP & 3 & 6 & 6 \\
\hline Flex. PIP & 3 & 6 & 6 \\
\hline Flex. both & 3 & 6 & 6 \\
\hline Power 65 mm & 6 & 6 & 9 \\
\hline Power 53 mm & 6 & 9 & 6 \\
\hline Power 39 mm & 6 & 6 & 9 \\
\hline Power 28 mm & 0 & 9 & 6 \\
\hline Tip pinch & 6 & 9 & 6 \\
\hline Writing tripod & 3 & 9 & 9 \\
\hline Pliers & 0 & 6 & 9 \\
\hline Catch bottle & 0 & 0 & 6 \\
\hline \hline Total & 36 & 72 & 78 \\
\hline
\end{tabular}

During the first session, the glued sensors sometimes detached from the user's finger, making it impossible to exploit the data for a number of tasks. Differences in repetitions between second and third sessions came from unusable data from the video tracking system due to missing markers trajectories, except for the bottle catching task that was added during the third session.

\section{Results}

1) Sensor response to finger flexion and extension: The sensitivities were calculated from the calibration runs as plotted Fig. 6:

- $>8 \times 10^{-4} / \mathrm{deg}$ in the $0 \mathrm{deg}$ to $25 \mathrm{deg}$ range and $>6 \times 10^{-3} / \mathrm{deg}$ for the $25 \mathrm{deg}$ to $60 \mathrm{deg}$ range for the MCP joint sensor

- $>1 \times 10^{-3} / \mathrm{deg}$ in the $12 \mathrm{deg}$ to $65 \mathrm{deg}$ range and $>7 \times 10^{-3} / \mathrm{deg}$ in the $65 \mathrm{deg}$ to $90 \mathrm{deg}$ range for the PIP joint sensor.

These sensitivities are equivalent to more than $5 \Omega / \mathrm{deg}$, both sensors having an initial resistance larger than 6.9 $\mathrm{k} \Omega$ (third data acquisition session). We also observed a hysteresis in the sensor response to joint's flexion and extension. The maximum width of the hysteresis loop corresponds to about $10 \mathrm{deg}$ for both sensors.

2) Quantitative comparison with the camera tracking system: After calibration, we compared the response of the sensor with the reference motion tracking system. Fig. 7

\section{TABLE II}

MEAN ABSOLUTE ERRORS AND BASELINE RESISTANCES THROUGH ALL TRIALS, WITH $100 \mu \mathrm{M}$ AND $50 \mu \mathrm{M}$ WIDE GAUGE TRACKS.

\begin{tabular}{|c||c|c|c|}
\hline & $100 \mu \mathrm{m}$, glue & $100 \mu \mathrm{m}$, tape & $50 \mu \mathrm{m}$, tape \\
\hline$\left\langle D_{M C P}\right\rangle \pm \sigma(\mathrm{deg})$ & $8.8 \pm 15$ & $7.4 \pm 5.2$ & $6.1 \pm 4.5$ \\
\hline$\left\langle D_{P I P}\right\rangle \pm \sigma(\mathrm{deg})$ & $11.6 \pm 8.7$ & $8.4 \pm 7.0$ & $8.3 \pm 7.0$ \\
\hline$\left\langle R_{0, M C P}\right\rangle \pm \sigma(\Omega)$ & $2591 \pm 29$ & $2321 \pm 107$ & $8137 \pm 179$ \\
\hline$\left\langle R_{0, P I P}\right\rangle \pm \sigma(\Omega)$ & $3020 \pm 19$ & $1970 \pm 37$ & $6912 \pm 122$ \\
\hline
\end{tabular}

(a)

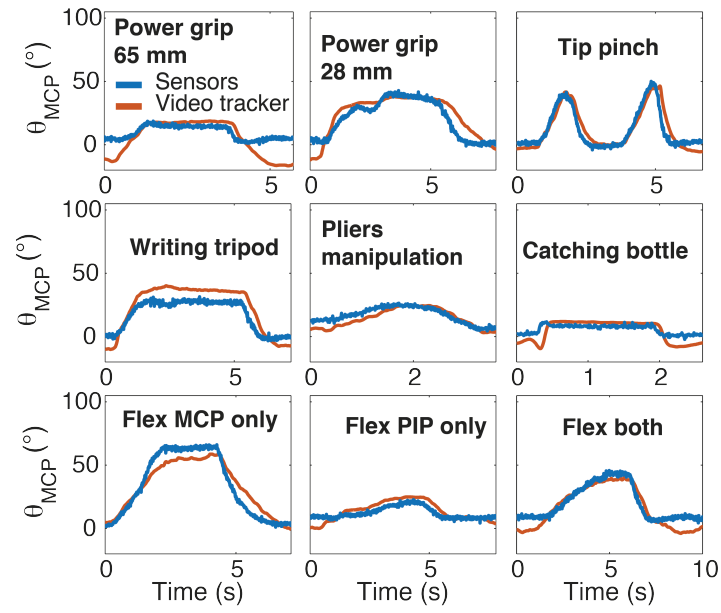

(b)

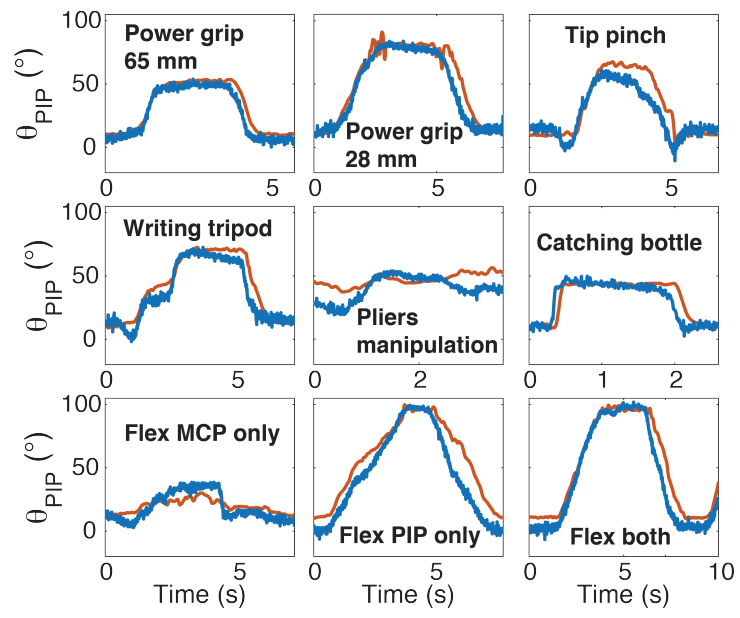

Fig. 7. Comparison between the index MCP (a) and PIP (b) angles sensed by the camera tracking system (orange) and by the soft skin-like sensor pair (blue) after calibration for 9 different tasks. A 10 points moving average filter was applied to the sensor's output, recorded at a $1 \mathrm{kHz}$ sampling rate.

shows the typical time response of the sensor for nine grasps and finger movements, representative of an entire session.

The absolute deviation D between our sensors and the reference was computed as:

$$
D=\left|\theta_{\text {sens }}-\theta_{\text {ref }}\right|
$$

where $\theta_{\text {sens }}$ is the angle computed from the response of the sensors' pair and $\theta_{\text {ref }}$ is the output of the reference motion camera tracking system.

Table II summarizes the results of all runs. The accuracy, defined as the average of $\mathrm{D}$ over all runs of a session after calibration, reached down to $6.1 \mathrm{deg}$ for the MCP angle and down to $8.3 \mathrm{deg}$ for the PIP angle across all tasks. We noted that eliminating the dead-zone by slightly pre-stretching the sensors when using the tape resulted in higher accuracy. The baseline of the sensors was also stable over the whole test session. No overshoot was observed, and the response of the sensor was fast, as depicted in the bottle catching 
task. Finally, we observed no significant improvement in accuracy when decreasing the meander track width from $100 \mu \mathrm{m}$ to $50 \mu \mathrm{m}$ to increase the strain gauges resistance. We propose that the accuracy of the sensors is ultimately limited by the width of the hysteresis loop. Mechanical crosstalk might also explain the lower accuracy in the PIP angle measurement.

\section{E. Recording on multiple fingers during a fine motor task}

We did not observe any impediment to the natural motion of fingers when a pianist was performing a short sample of Beethoven Sonata 24 for Piano while wearing the sensors (Fig. 1 and supplementary video). All sensors remained functional and barely noticed by the wearer during a 30 minute session. This demonstration showcases the high sensitivity and well defined dynamic range of our wearable epidermal sensors.

\section{CONCLUSION}

We presented resistive strain gauges produced by a novel metallization technology based on intrinsically stretchable biphasic thin metal films deposited and micro-structured on elastomeric substrates. These rubber-like gauges are soft (Young's modulus $<2 \mathrm{MPa}$ ), with thickness approaching that of the outermost layer of the human skin $(<50 \mu \mathrm{m})$. One single gauge is 24 times more sensitive to large (50 $\%)$ axial strain than transverse strain, and quasi-insensitive to normal pressure up to $100 \mathrm{kPa}$. We proposed a design integrating a pair of sensors to encode the flexion and extension of human fingers. We demonstrated that our sensors have a fast and stable response, with more than $10^{\circ}$ accuracy when compared to a commercial vision based tracking system. The performance was maintained through a wide range of repeated dexterous manipulation tasks. We finally mounted three pairs of sensors on a human hand and recorded the sensors' output when performing a fine motor task, showing the minimal impact of our sensing system on the user's dexterity. The extensive characterization and demonstrated stability of the soft sensors let us envision a translation to robotic and therapeutic applications. Beyond their accuracy our sensors fulfill the requirements for truly skin-conforming motion sensing: they are unobtrusive, adjustable and can be integrated in a portable system. As a next step, we will optimize the design of our sensors to encode the thumb movement, which has a more complex range of motion due to its saddle joint. A calibration scheme involving no external reference system will also be investigated. In this way, we will be able to fully track the human hand movements during manipulation and recalibrate the sensors when needed. Our soft technology could then be deployed for neurorehabilitation medicine and closed-loop control of assistive devices such as hand exoskeletons.

\section{ACKNOWLEDGMENTS}

We acknowledge M. Coscia and S. Micera (TNE-EPFL) for providing training and help with the Vicon system, and C. Tringides (LSBI-EPFL) for her time and piano skills.

\section{REFERENCES}

[1] H. Zhou and H. Hu, "Human motion tracking for rehabilitation - A survey," Biomedical Signal Processing and Control, vol. 3, no. 1, pp. $1-18,2008$.

[2] H. Zhou, T. Stone, H. Hu, and N. Harris, "Use of multiple wearable inertial sensors in upper limb motion tracking," Medical engineering \& physics, vol. 30, no. 1, pp. 123-133, 2008.

[3] C.-Y. Park, J.-H. Bae, and I. Moon, "Development of wireless data glove for unrestricted upper-extremity rehabilitation system," 2009 Iccas-Sice, pp. 790-793, 2009.

[4] H. Chen, Q. Wang, and L. Cao, "Design of the workstation for hand rehabilitation based on data glove," 2010 IEEE International Conference on Bioinformatics and Biomedicine Workshops, BIBMW 2010, pp. 769-771, 2010.

[5] M. Amjadi, K.-U. Kyung, I. Park, and M. Sitti, "Stretchable, SkinMountable, and Wearable Strain Sensors and Their Potential Applications: A Review," Advanced Functional Materials, 2016.

[6] R. A. Bilodeau, E. L. White, and R. K. Kramer, "Monolithic Fabrication of Sensors and Actuators in a Soft Robotic Gripper," in 2015 IEEE/RSJ International Conference on Intelligent Robots and Systems (IROS), pp. 2324-2329.

[7] F. Amirabdollahian, S. Ates, A. Basteris, A. Cesario, J. Buurke, H. Hermens, D. Hofs, E. Johansson, G. Mountain, N. Nasr, S. Nijenhuis, G. Prange, N. Rahman, P. Sale, F. Schätzlein, B. van Schooten, and A. Stienen, "Design, development and deployment of a hand/wrist exoskeleton for home-based rehabilitation after stroke - SCRIPT project," Robotica, vol. 32, no. 8, pp. 1331-1346, 2014.

[8] P. Polygerinos, Z. Wang, K. C. Galloway, R. J. Wood, and C. J. Walsh, "Soft robotic glove for combined assistance and at-home rehabilitation," Robotics and Autonomous Systems, vol. 73, pp. 135$143,2014$.

[9] A. P. Gerratt, H. O. Michaud, and S. P. Lacour, "Elastomeric Electronic Skin for Prosthetic Tactile Sensation," Advanced Functional Materials, vol. 25, pp. 2287-2295, 2015.

[10] H. O. Michaud, J. Teixidor, and S. P. Lacour, "Soft Flexion Sensors Integrating Stretchable Metal Conductors on a Silicone Substrate for Smart Glove Applications," in 2015 IEEE International Conference on Micro Electro Mechanical Systems, pp. 760-763.

[11] J.-B. Chossat, Y. Tao, V. Duchaine, and Y.-L. Park, "Wearable Soft Artificial Skin for Hand Motion Detection with Embedded Microfluidic Strain Sensing," in 2015 IEEE International Conference on Robotics and Automation (ICRA), pp. 2568-2573.

[12] N. Lu, C. Lu, S. Yang, and J. Rogers, "Highly Sensitive SkinMountable Strain Gauges Based Entirely on Elastomers," Advanced Functional Materials, vol. 22, no. 19, pp. 4044-4050, 2012.

[13] R. K. Kramer, C. Majidi, R. Sahai, and R. J. Wood, "Soft curvature sensors for joint angle proprioception," in 2011 IEEE/RSJ International Conference on Intelligent Robots and Systems (IROS), pp. 19191926.

[14] F. Hammond, Y. Mengüç, and R. J. Wood, "Toward a modular soft sensor-embedded glove for human hand motion and tactile pressure measurement," in 2014 IEEE/RSJ International Conference on Intelligent Robots and Systems (IROS), pp. 4000-4007.

[15] A. Hirsch, H. O. Michaud, A. P. Gerratt, S. de Mulatier, and S. P. Lacour, "Intrinsically Stretchable Biphasic (Solid-Liquid) Thin Metal Films," Advanced Materials, 2016.

[16] O. Kuwazuru, J. Saothong, and N. Yoshikawa, "Mechanical approach to aging and wrinkling of human facial skin based on the multistage buckling theory," Medical Engineering \& Physics, vol. 30, no. 4, pp. $516-522,2008$

[17] A. Buryanov and V. Kotiuk, "Proportions of Hand Segments," International Journal of Morphology, vol. 28, no. 3, pp. 755-758, 2010.

[18] M. Mentzel, A. Benlic, N. J. Wachter, D. Gulkin, S. Bauknecht, and J. Glke, "The Dynamics of Motion Sequences of the Finger Joints during Fist Closure," Handchir Mikrochir Plast Chir, vol. 43, no. 3, pp. 147-154, 2011.

[19] R. B. Watson, in Springer handbook of experimental solid mechanics, W. N. Sharpe Jr and W. N. Sharpe, Eds. Springer, 2008, pp. 283-333.

[20] C. D. Metcalf, S. V. Notley, P. H. Chappell, J. H. Burridge, and V. T. Yule, "Validation and application of a computational model for wrist and hand movements using surface markers," IEEE Transactions on Biomedical Engineering, vol. 55, no. 3, pp. 1199-1210, 2008. 